\title{
VaR-Optimal Risk Management in Regime-Switching Jump-Diffusion Models
}

\author{
Alessandro Ramponi \\ Department of Economics and Finance, University of Roma Tor Vergata, Roma, Italy \\ Email: ramponi@economia.uniroma2.it \\ Received October 6, 2012; revised November 13, 2012; accepted November 26, 2012
}

\begin{abstract}
In this paper we study a classical option-based portfolio strategy which minimizes the Value-at-Risk of the hedged position in a continuous time, regime-switching jump-diffusion market, by using Fourier Transform methods. However, the analysis of this hedging strategy, as well as the computational technique for its implementation, is fairly general, i.e. it can be applied to any dynamical model for which Fourier transform methods are viable.
\end{abstract}

Keywords: Regime Switching Jump-Diffusion Models; Value at Risk; Risk Management; Fourier Transform Methods

\section{Introduction}

In this paper we study a classical hedging policy based on options followed by an institutional manager whose aim is to minimize the Value-at-Risk of a position in a regime-switching jump-diffusion market. Although sharply criticized for the lack of sub-additivity and its inability to quantify the severity of an exposure to rare events, VaR has been adopted as a benchmark in the financial industry and for regulatory purposes. It plays a central role in banking regulation and internal risk management, mainly due to its simplicity. The analysis of this hedging strategy has been initiated by [1] a decade ago for a portfolio made by a risky asset following a lognormal random dynamic, and hence analytically solved in a Black-Scholes setting. More recently, it has been considered for a bond portfolio in $[2,3]$. By taking the VaR as the risk measure for potential losses $L$ of a portfolio, we hedge the risky position by buying a fraction $h$ of a put option with maturity $T$ and strike price $K$ : but what $K$ and $h$ ? By fixing a hedging constraint, the corresponding (constrained) optimality condition involves quantiles computations and derivative pricing. Both steps can be efficiently faced with the Fourier transform technique, under historical and risk-neutral probability respectively.

The dynamic model we consider for the risky position is $S(t)=S_{0} \mathrm{e}^{X(t)}$, where $X(t)$ is specified on a filtered probability space as a jump-diffusion whose parameters change over time, driven by a continuous time and stationary Markov Chain on a finite state space $\mathcal{S}$, representing the unobserved state of the world. In fact, empirical studies on the behavior of financial markets show the ability of regime-switching models to capture some peculiarities in the observed data, as firstly highlighted in the seminal paper by Hamilton [4]. Since then, there has been a growing effort in applying switching models to a wide class of financial and/or economic problems. On the other hand, the necessity of including jumps in the underlying models to provide better representation of their dynamical properties is widely recognized (see e.g. [5]). Empirical stylized facts about observed data, such as volatility clustering and heavy tails, are then well captured by regime-switching jumpdiffusions which turns out to be an appealing and flexible class of dynamic models. The computation of quantiles in regime-switching models has been considered by several authors mainly in discrete-time setting (see e.g. [6,7] and ref. therein). Here we consider this problem in the continuous time framework in which the required computations can be very efficiently implemented with the help of Fourier Transform methods (see e.g. [8]). The use of this kind of technique for the analytical calculation of VaR has been considered in Duffie and Pan [9] in terms of the Fourier inversion of the characteristic function. The use of Generalized Fourier Transform and the FFT algorithm is more recent: see Le Courtois and Walter [10], Kim et al. [11] and Scherer et al. [12].

The paper is organized as follows: we firstly derive the optimality conditions for the VaR minimizing strategy (Section 2) and then (Section 3) we introduce the regimeswitching dynamic model, its generalized characteristic function and the change-of-measure result for switching from the historical to the risk-neutral probability. Finally, in Section 4 we specify the Fourier Transform technique for calculating quantiles and put/call option prices and 
report some numerical experiments to show the impact of jumps and regime-switching on the optimal hedging strategy.

Some final comments can be briefly outlined. Firstly, the analysis of the hedging strategy is fairly general, that is it can be applied to any dynamical model for which Fourier transform methods are viable, for example it can be extended to Variance-Gamma or Bates models. Furthermore, besides the choice of different dynamic models, it would be interesting to consider alternative risk measures, such as the Conditional Value at Risk (CVaR). This is certainly less commonly used in finance industry, but it is widely used in insurance industry being a coherent, convex and stable risk measure (see [13]).

\section{VaR and Optimal Risk Management}

Given a confidence level $\alpha \in(0,1)$, the set of $\epsilon$-quantiles of the random variable $Y$ is the interval $\left[q_{\alpha}^{-}(Y), q_{\alpha}^{+}(Y)\right]$ where

$q_{\alpha}^{-}(Y)=\inf \{q \in \mathbb{R} \mid P(Y \leq q) \geq \alpha\}$ and

$q_{\alpha}^{+}(Y)=\inf \{q \in \mathbb{R} \mid P(Y \leq q)>\alpha\}$. For a random variable having continuous and strictly increasing distributions function $F_{Y}(y)$,

$q_{\alpha}^{-}(Y)=q_{\alpha}^{+}(Y) \equiv q_{\alpha}(Y)=F_{Y}^{-1}(\alpha)$, i.e. it solves the equation $\mathbb{P}\left(Y \leq q_{\alpha}(Y)\right)=\alpha$.

Here we take the portfolio loss $L$ to describe a financial position in a fixed time interval and, in order to simplify notations, we assume in this section that $L$ has a continuous and strictly increasing distributions function. The Value-at-Risk at level $\alpha$ is defined as

$$
\operatorname{VaR}_{\alpha}(L) \equiv \inf \{q \in \mathbb{R} \mid P(L \leq q)>\alpha\} .
$$

Let $S_{t}$ be the value of the risky asset, $t \in[0, T]$ and $r$ be the risk-free rate, that without loss of generality we consider fixed in the period: we define the loss at time 0 of such a position as $L^{u}=S_{0}-\mathrm{e}^{-r T} S_{T}$ implying $\operatorname{VaR}_{\alpha}\left(L^{u}\right)=S_{0}-\mathrm{e}^{-r T} q_{1-\alpha}\left(S_{T}\right)$. Let us now consider a classical hedging problem in which an institution has an exposure to a risky asset $S_{t}$ and decide to hedge such an exposure in the interval $[0, T]$ by buying a fraction $h \in[0,1]$ of an European put option on the asset with maturity $T$ and strike price $K$. Analogously to the situation considered in [1], we take as the hedged position the portfolio composed by the risky asset and the put option: the loss of the hedged portfolio at time 0 is therefore $L^{h, K}=S_{0}+h \Pi_{0}^{P}(K, T)-\mathrm{e}^{-r T}\left(S_{T}+h\left(K-S_{T}\right)^{+}\right)$,

where $\Pi_{t}^{P}(K, T)$ is the price of the put option at time $t$. By defining the strictly increasing function

$g(u)=u-h(u-\bar{K})^{+}+h \Pi_{0}^{P}(K, T)$, where

$\bar{K}=S_{0}-\mathrm{e}^{-r T} K$, it is immediately seen that
$L^{h, K}=g\left(L^{u}\right) ;$ therefore

$\operatorname{VaR}_{\alpha}\left(L^{h, K}\right)=g\left(\operatorname{VaR}_{\alpha}\left(L^{u}\right)\right)=\operatorname{VaR}_{\alpha}\left(L^{u}\right)+h \Pi_{0}^{P}(K, T)$

$-\mathrm{e}^{-r T} h\left(K-q_{1-\alpha}\left(S_{T}\right)\right)^{+}$.

Let us firstly notice that if $K \leq q_{1-\alpha}\left(S_{T}\right)$, then $\operatorname{VaR}_{\alpha}\left(L^{h, K}\right)>\operatorname{VaR}_{\alpha}\left(L^{u}\right)$ since $\Pi_{0}^{P}(K, T)>0$. Hence, given the budget constraint $C$, the optimal hedging strategy is specified by the following problem:

$$
\left\{\begin{array}{l}
\min _{K, h}\left\{\operatorname{VaR}_{\alpha}\left(L^{u}\right)+h \Pi_{0}^{P}(K, T)-\mathrm{e}^{-r T} h\left(K-q_{1-\alpha}\left(S_{T}\right)\right)\right\} \\
h \Pi_{0}^{P}(K, T)=C, \\
h \in(0,1], K>q_{1-\alpha}\left(S_{T}\right) .
\end{array}\right.
$$

Since $h=C / \Pi_{0}^{P}(K, T)$, the optimality first order condition for $K$ is given by the following non-linear equation:

$$
\Pi_{0}^{P}(K, T)=\left(K-q_{1-\alpha}\left(S_{T}\right)\right) \frac{\partial}{\partial K} \Pi_{0}^{P}(K, T) .
$$

Assuming that (2) has a solution $K^{*}>q_{1-\alpha}\left(S_{T}\right)$ and the twice differentiability of the price functional we can prove that this is actually a minimum since

$$
\begin{aligned}
& \frac{\partial^{2} \operatorname{VaR}_{\alpha}\left(L^{h, K}\right)}{\partial K^{2}} \\
& =\frac{\mathrm{e}^{-r T} C\left(K^{*}-q_{1-\alpha}\left(S_{T}\right)\right)}{\Pi^{P}\left(K^{*}, T\right)^{2}} \times \frac{\partial^{2} \Pi^{P}}{\partial K^{2}}\left(K^{*}, T\right)>0
\end{aligned}
$$

by the convexity of the price functional w.r.t. the strike. Correspondingly, the optimal amount of the hedging put option is

$$
h^{*}=\frac{C}{\Pi_{0}^{P}\left(K^{*}, T\right)} .
$$

We now assume the following:

Assumption 2.1. The price of the put option can be represented as the discounted expected value of the payoff at time $T$ under a risk-neutral measure $\mathbb{Q}$ :

$$
\Pi_{t}^{P}(K, T)=\mathrm{e}^{-r T} \mathbb{E}^{\mathbb{Q}}\left[\left(K-S_{T}\right)^{+}\right] .
$$

Furthermore, let $F_{S}(s)=\mathbb{Q}\left(S_{T} \leq s\right)$ be the cumulative distribution function (cdf) of the random variable $S_{T}$ under such a measure: hence

$$
\begin{aligned}
\Pi_{t}^{P}(K, T) & =\mathrm{e}^{-r T} \int_{0}^{+\infty}(K-s)^{+} \mathrm{d} F_{S}(s) \\
& =\mathrm{e}^{-r T}\left(K \mathbb{Q}\left(S_{T} \leq K\right)-\int_{0}^{K} s \mathrm{~d} F_{S}(s)\right)
\end{aligned}
$$

and 


$$
\frac{\partial}{\partial K} \Pi_{t}^{P}(K, T)=\mathrm{e}^{-r T} \mathbb{Q}\left(S_{T} \leq K\right) .
$$

We can finally prove the following property:

Proposition 2.1. If $K^{*}>q_{1-\alpha}\left(S_{T}\right)$, then

$\operatorname{VaR}_{\alpha}\left(L_{0}^{h^{*}, K^{*}}\right)<\operatorname{VaR}_{\alpha}\left(L^{u}\right)$.

Proof. Since $K^{*}$ and $h^{*}$ are characterized through (2) and (3), we get

$$
\begin{aligned}
& \operatorname{VaR}_{\alpha}\left(L_{0}^{h^{*}, K^{*}}\right) \\
= & \operatorname{VaR}_{\alpha}\left(L^{u}\right) \\
& +\frac{C}{\Pi_{t}^{P}\left(K^{*}, T\right)}\left(\Pi_{t}^{P}\left(K^{*}, T\right)-\mathrm{e}^{-r T}\left(K^{*}-q_{1-\alpha}\left(S_{T}\right)\right)\right) \\
= & \operatorname{VaR}_{\alpha}\left(L^{u}\right) \\
& +\frac{C}{\Pi_{t}^{P}\left(K^{*}, T\right)}\left(K^{*}-q_{1-\alpha}\left(S_{T}\right)\right)\left(\frac{\partial}{\partial K} \Pi_{t}^{P}\left(K^{*}, T\right)-\mathrm{e}^{-r T}\right) .
\end{aligned}
$$

From Assumption 2.1, we have

$$
\frac{\partial}{\partial K} \Pi_{t}^{P}(K, T)-\mathrm{e}^{-r T}=\mathrm{e}^{-r T}\left(\mathbb{Q}\left(S_{T} \leq K\right)-1\right)<0 .
$$

Therefore

$$
\begin{aligned}
& \operatorname{VaR}_{\alpha}\left(L_{0}^{h^{*}, K^{*}}\right)=\operatorname{VaR}_{\alpha}\left(L^{u}\right) \\
& +\frac{C}{\Pi_{t}^{P}\left(K^{*}, T\right)}\left(K^{*}-q_{1-\alpha}\left(S_{T}\right)\right) \mathrm{e}^{-r T}\left(\mathbb{Q}\left(S_{T} \leq K^{*}\right)-1\right) \\
& <\operatorname{VaR}_{\alpha}\left(L^{u}\right) .
\end{aligned}
$$

Remark 2.1. Notice that the optimality condition (2) under Assumption 2.1 simplifies to

$$
\frac{1}{\mathbb{Q}\left(S_{T} \leq K\right)} \int_{-\infty}^{K} s \mathrm{~d} F_{S}^{Q}(x)=q_{1-\alpha}\left(S_{T}\right)
$$

and depends on both the objective and the risk neutral distributions $\mathbb{P}$ and $\mathbb{Q}$. Furthermore, it easily seen that the 1.h.s. is equal to the conditional expectation $\mathbb{E}^{Q}\left[S_{T} \mid S_{T} \leq K\right]$ which is an increasing function of $K$ bounded by $\mathbb{E}^{\mathbb{Q}}\left[S_{T}\right]$. Therefore, (4) has a unique solution if and only if $q_{1-\alpha}\left(S_{T}\right)<\mathbb{E}^{\mathbb{Q}}\left[S_{T}\right]$.

\section{Regime-Switching Jump Diffusions and Measure Change}

Let us consider on a filtered probability space $\left(\Omega, \mathcal{F}, \mathcal{F}_{t}, \mathbb{P}\right)$ a stochastic process of the form $S_{t}=S_{0} \mathrm{e}^{X_{t}}, S_{0}>0$, modeling the value, of a risky asset for $t \in[0, T]$. We consider a jump-diffusion setting in which the jump process is described as a marked point process (MPP), that is a random measure $p(d y, d t)$ characterized by the intensity process

$\lambda_{t}(d y)$, the parameters of which are driven by a finite state and continuous time Markov chain. So, let $\alpha(t)$ be a continuous time, homogeneous and stationary Markov Chain on the state space $\mathcal{S}=\{1,2, \cdots, M\}$ with generator $H \in \mathbb{R}^{M \times M}$ : the elements $h_{i j}$ of the matrix $H$ are positive numbers such that $\sum_{j \neq i, j=1}^{M} h_{i j}=-h_{i i}$, for $i=1, \cdots, M$. Furthermore, $\mu: \mathcal{S} \rightarrow \mathbb{R}, \sigma: \mathcal{S} \rightarrow \mathbb{R}$ and $\gamma: E \times \mathcal{S} \rightarrow \mathbb{R}$ are given functions, $(E, \mathcal{E})$ being the measurable mark space. Without loss of generality, we can assume in the following $E \subseteq \mathbb{R}$. In a given interval $0 \leq t \leq T$, we consider the dynamic

$$
\begin{aligned}
& d X(t)=\left(\mu(\alpha(t))-\frac{1}{2} \sigma^{2}(\alpha(t))\right) d t+\sigma(\alpha(t)) d W(t) \\
& +\int_{E} \gamma\left(y, \alpha\left(t^{-}\right)\right) p^{\alpha}(d y, d t), X(0)=0,
\end{aligned}
$$

where $W(t)$ is a standard brownian motion and $p^{\alpha}(d y, d t)$ is a MPP characterized by the intensity $\lambda_{t}^{\alpha}(d y) \equiv \lambda(\alpha) m(\alpha, d y)$. Here $\lambda(\cdot)$ represents the (regime-switching) intensity of the Poisson process $N_{t}$, while $m(\cdot, d y)$ are a set of probability measures on $E$, one for each state (regime) $i \in \mathcal{S}$. The function $\gamma(y, \alpha)$ represents the jump amplitude relative to the mark $y$ in regime $\alpha$. The couple $(\lambda(\alpha), m(\alpha, d y))$ is called the $\left(\mathbb{P}, \mathcal{F}_{t}\right)$-local characteristic of $p^{\alpha}(d s, d y)$.

Furthermore $\int_{0}^{t} \int_{E} \mathcal{H}(y, s)\left(p^{\alpha}(d y, d s)-\lambda_{s}^{\alpha}(d y) d s\right)$ is a martingale for a suitable class of processes $\mathcal{H}$ (see [14]),

$$
q^{\alpha}(d y, d t)=p^{\alpha}(d y, d t)-\lambda\left(\alpha\left(t^{-}\right)\right) m\left(\alpha\left(t^{-}\right), d y\right) d t
$$

being the compensated process.

Throughout the paper we assume that the processes $\alpha(\cdot)$ and $W(\cdot)$ are independent, $W(\cdot)$ and $p^{\alpha}(d y, d t)$ are conditionally independent given $\alpha(t)$ and that $\mathbb{E}\left[\mathrm{e}^{\gamma(Y, \alpha)}\right]=\int_{E} \mathrm{e}^{\gamma(y, \alpha)} m(\alpha, d y)$ is finite for each regime $\alpha$. An application of the generalized Ito's Formula gives the corresponding jump-diffusion SDE for the asset price

$$
\begin{aligned}
& \frac{d S(t)}{S\left(t^{-}\right)} \\
& =\mu(\alpha(t)) d t+\sigma(\alpha(t)) d W(t) \\
& +\int_{E}\left(\mathrm{e}^{\gamma(y, \alpha(t-))}-1\right) p^{\alpha}(d y, d t) \\
& =[\mu(\alpha(t))+\lambda(\alpha(t)) \kappa(\alpha(t))] d t \\
& +\sigma(\alpha(t)) d W(t)+\int_{E}\left(\mathrm{e}^{\gamma(y, \alpha(t-))}-1\right) q^{\alpha}(d y, d t),
\end{aligned}
$$

with $S(0)=S_{0}$ and $\kappa(\alpha)=\mathbb{E}\left[\mathrm{e}^{\gamma(Y, \alpha)}-1\right]$.

Measure changes. An absolutely continuous transformation of measures in a jump-diffusion setting allows 
to change the intensities of the MPP and the Markov chain in addition to the translation of the Wiener process (see [14]). It results convenient to represent the underlying Markov chain itself as the MPP $v(d t, d z)$ with finite mark space $(\mathrm{S}, \mathcal{P}(\mathrm{S}))$,

$\mathrm{S}=\{z=(i, j): i \neq j, i, j \in\{1,2, \cdots, M\}\}$ and $\mathcal{P}(\mathrm{S})=2^{\mathrm{S}}$ : the compensator is

$$
\lambda_{v}(\alpha(t-), d z)=\sum_{i \neq j} h_{i j} \mathbb{I}_{\{\alpha(t-)=i\}} \epsilon_{(i, j)}(d z)
$$

$\epsilon_{(i, j)}(d z)$ being the Dirac measure. Consequently, let

$\vartheta_{t}(\alpha)$ be a square integrable predictable processes,

$h_{t}(\alpha, y)$ a non-negative function such that $\int_{E} h_{t}(\alpha, y) m(\alpha, d y)=1, \forall t \in[0, T]$ and let $\psi(\alpha)$ and $\Phi(z)$ be strictly positive functions defined on $\mathcal{S}$ and $\mathrm{S}$, respectively. We can define a new measure $\mathbb{Q}$ on the measurable space by setting

$$
\left\{\begin{array}{l}
d W_{t}^{Q}=d W_{t}-\vartheta_{t}(\alpha(t)) d t \\
q^{Q, \alpha}(d t, d y)=p(d t, d y)-\psi(\alpha(t)) \lambda(\alpha(t)) h_{t}(\alpha(t), y) m(\alpha(t), d y) d t \\
\lambda_{v}^{Q}(\alpha, d z)=\Phi(z) \lambda_{v}(\alpha, d z) .
\end{array}\right.
$$

Besides the translation of the Wiener process $W_{t}$, we perform a change in the intensity of the MPP giving the compensated process $q^{Q, \alpha}(d t, d y)$ with $\left(\mathbb{Q}, \mathcal{F}_{t}\right)$-local characteristic $\left(\psi(\alpha(t)) \lambda(\alpha(t)), h_{t}(\alpha(t), y) m(\alpha(t), d y)\right)$ and a change of the intensity of the Markov chain which under $\mathbb{Q}$ has generator $H^{Q}=\left\{h_{i j}^{Q}\right\}$ where $h_{i j}^{Q}=\Phi(i, j) h_{i j}, \quad h_{i i}^{Q}=-\sum_{k=1, k \neq i}^{M} \Phi(i, k) h_{i k}, \quad i=1, \cdots, M$.

By taking the Radon-Nikodym derivative

$$
\begin{aligned}
L_{t}= & \exp \left(-\frac{1}{2} \int_{0}^{t} \vartheta_{s}(\alpha(s))^{2} \mathrm{~d} s+\int_{0}^{t} \vartheta_{s}(\alpha(s)) \mathrm{d} W_{s}^{Q}+\int_{0}^{t}(1-\psi(\alpha(s)) \lambda(\alpha(s))) \mathrm{d} s\right. \\
& \left.+\int_{0}^{t} \int_{E} \log \left(\psi(\alpha(s)) h_{t}(\alpha(s), y)\right) p(d s, d y)+\int_{0}^{t} \int_{S}(1-\Phi(z)) \lambda_{v}(\alpha(t-), d z) \mathrm{d} s+\int_{0}^{t} \int_{S} \log (\Phi(z)) v(d s, d z)\right)
\end{aligned}
$$

and supposing that $\mathbb{E}^{\mathbb{P}}\left[L_{t}\right]=1$ for $t \in[0, T]$, we have a $d \mathbb{Q}=L_{T} d \mathbb{P}$, under which probability measure $\mathbb{Q}$ on $\mathcal{F}$ equivalent to $\mathbb{P}$ with

$$
\begin{aligned}
& \frac{d S(t)}{S\left(t^{-}\right)}=\left[\mu(\alpha(t))+\sigma(\alpha(t)) \vartheta_{t}(\alpha(t))+\psi(\alpha(t)) \lambda(\alpha(t)) \kappa^{Q}(\alpha(t))\right] d t \\
& +\sigma(\alpha(t)) d W^{Q}(t)+\int_{E}\left(\mathrm{e}^{\gamma(y, \alpha(t-))}-1\right) q^{Q, \alpha}(d y, d t),
\end{aligned}
$$

where $\kappa^{Q}(\alpha)=\mathbb{E}^{\mathbb{Q}}\left[\mathrm{e}^{\gamma(Y, \alpha)}-1\right]$ (see [14]).

In order to price derivatives under the model (6) we need to specify a risk-neutral or martingale measure, that is a measure under which the discounted price process $\mathrm{e}^{-r t} S_{t}$ is a martingale. This is done by taking

$$
\vartheta(\alpha) \equiv \frac{r-\mu(\alpha)-\psi(\alpha) \lambda(\alpha) \kappa^{Q}(\alpha)}{\sigma(\alpha)}
$$

from which we finally get the risk-neutral dynamic for the underlying

$$
\begin{aligned}
& \frac{d S(t)}{S\left(t^{-}\right)}=r d t+\sigma(\alpha(t)) d W^{Q}(t) \\
& +\int_{E}\left(\mathrm{e}^{\gamma(y, \alpha(t-))}-1\right) q^{Q, \alpha}(d y, d t) \\
& =\left[r-\psi(\alpha(t)) \lambda(\alpha(t)) \kappa^{Q}(\alpha(t))\right] d t \\
& +\sigma(\alpha(t)) d W^{Q}(t)+\int_{E}\left(\mathrm{e}^{\gamma(y, \alpha(t-))}-1\right) p^{Q, \alpha}(d y, d t) .
\end{aligned}
$$

Correspondingly, for the process $X(t)$ we have

$$
\begin{aligned}
& d X(t) \\
= & {\left[r-\frac{1}{2} \sigma^{2}(\alpha(t))\right] d t+\sigma(\alpha(t)) d W^{Q}(t) } \\
+ & \int_{E} \gamma\left(y, \alpha\left(t^{-}\right)\right) q^{Q, \alpha}(d y, d t) \\
= & {\left[r-\frac{1}{2} \sigma^{2}(\alpha(t))-\psi(\alpha(t)) \lambda(\alpha(t)) \kappa^{Q}(\alpha(t))\right] d t } \\
+ & \sigma(\alpha(t)) d W^{Q}(t) \\
+ & \int_{E} \gamma\left(y, \alpha\left(t^{-}\right)\right) p^{Q, \alpha}(d y, d t) .
\end{aligned}
$$

The measure transformation defined by (8) through (9) preserves the probability structure of the stochastic process $X(t)$ under both $\mathbb{P}$ and $\mathbb{Q}$. It worth noting that we can specify infinitely many equivalent measures $\mathbb{Q}$. In practice, the usual way to select one of the equivalent 
measures is to calibrate the model to a set of observed data.

GFT for regime-switching jump-diffusions. In order to apply Fourier methods, we need to calculate the Fourier transform $\phi_{X}(z)$ of our process. Since we have to consider the process $X_{T}$ under two different measure, we derive its characteristic function for the following general dynamic

$$
\begin{aligned}
& d X(t)=\xi(\alpha(t)) d t+\sigma(\alpha(t)) d W(t) \\
& +\int_{E} \gamma\left(y, \alpha\left(t^{-}\right)\right) p(d y, d t)
\end{aligned}
$$

with $(\bar{\lambda}(\alpha), \bar{m}(\alpha, d y))$ local characteristic, and Markov chain generator $Q$. In [8] it was proved the following

Proposition 3.1. Let $\phi_{j}(z)=\mathbb{E}\left[\mathrm{e}^{\mathrm{i} z \gamma(Y(j), j)}\right]$ be the generalized Fourier transform of the jump magnitude under the given measure. Then, by letting

$$
\vartheta_{j}(z)=\mathrm{z} \xi(j)+\frac{1}{2} \mathrm{i} z^{2} \sigma^{2}(j)-\mathrm{i} \bar{\lambda}(j)\left(\phi_{j}(z)-1\right)
$$

and $\tilde{\vartheta}_{i}(z)=\vartheta_{j}(z)-\vartheta_{M}(z)$, we have

$$
\begin{aligned}
\phi_{T}(z) & =\mathrm{e}^{\mathrm{i} \vartheta_{M}(z) T}\left(\mathbf{1}^{\prime} \cdot \mathrm{e}^{\left(Q^{\prime}+\mathrm{i} \operatorname{diag}\left(\tilde{\vartheta}_{1}(z), \cdots, \tilde{\vartheta}_{M-1}(z), 0\right)\right) T} \cdot \mathbb{I}(0)\right) \\
& =\mathbf{1}^{\prime} \cdot \mathrm{e}^{\left(Q^{\prime}+\mathrm{i} \operatorname{diag}\left(\tilde{\vartheta}_{1}(z), \cdots, \tilde{\vartheta}_{M-1}(z)\right)\right) T} \cdot \mathbb{I}(0),
\end{aligned}
$$

where $\mathbf{1}=(1, \cdots, 1)^{\prime} \in \mathbb{R}^{M \times 1}$,

$\mathbb{I}(0)=\left(\mathbb{I}_{\alpha(0)=1}, \cdots, \mathbb{I}_{\alpha(0)=M}\right)^{\prime} \in \mathbb{I}^{M \times 1}$ and $Q^{\prime}$ is the transpose of $Q$.

Different models can be recovered with simple linear constraints on the full parameter set of our model (RSJDM) (6), (12), $\xi_{i}, \sigma_{i}, \lambda_{i}, h_{i j}, i, j=1, \cdots, M$. This follows by noticing that if $\xi(i)=\xi, \sigma(i)=\sigma$,

$\lambda(i)=\lambda$ and $\phi_{i}(z)=\phi(z)$ we are implicitly assuming a unique regime so recovering the well-known characteristic function of the (single-regime) jump-diffusion

dynamic $\phi_{\Gamma}(z)=\exp \left(z \xi+\frac{1}{2} \mathrm{iz}^{2} \sigma^{2}-\mathrm{i} \lambda(\phi(z)-1)\right)$

which includes the standard geometrical Brownian motion (GBM) $(\lambda=0)$, the Merton jump-diffusion models (JDM); furthermore, if $\lambda_{i}=0$ we get the regimeswitching version of GBM (RSGBM).

The evaluation of the characteristic function requires to compute matrix exponentials for which efficient numerical techniques are available; conversely, the case $M=2$ can be considered explicitly (see [8] and the references therein).

\section{Computing Results}

In order to implement the optimal hedging strategy, we need to evaluate the VaR of the risky asset and the value of a put option. Both steps can be efficiently faced by means of Fourier methods. In this section we firstly outline such a technique and then we apply it to study numerically a model with two regimes and gaussian jumps.

\subsection{Fourier Methods}

Fourier transform methods are efficient techniques emerged in recent years as one of the main methodology for the evaluation of derivatives. Here we consider the technique introduced in [15] which consider the generalized Fourier transform with respect to the trigger parameter characterizing the payoff.

More formally, let $\Pi(S, K)$ be the payoff at maturity of the derivative: for example, $\Pi(S, K)=(K-S)^{+}$ is the payoff of the put option. The no-arbitrage price is therefore given by

$$
\Pi_{0}=\mathrm{e}^{-r T} \mathbb{E}^{\mathbb{Q}}\left[\Pi\left(S_{T}, K\right)\right] .
$$

Due to the exponential structure of the underlying dynamic $S_{t}=S_{0} \exp \left(X_{t}\right)$, it is convenient to represent the payoff with respect to the new variables

$\log (S)=X+\log \left(S_{0}\right) \equiv x$ and $\log (K) \equiv k$, in such a way

$$
\Pi(S, K)=\Pi\left(\exp \left(X+\log \left(S_{0}\right)\right), \exp (k)\right) \equiv \Pi(x, k) .
$$

Therefore, let us denote with $\Pi(x, k)$ an arbitrary payoff function and with $\hat{\Pi}_{x}(z)$ its generalized Fourier transform (GFT) w.r.t. $k$, that is

$$
\hat{\Pi}_{x}(z)=\int_{\mathbb{R}} \mathrm{e}^{\mathrm{i} z k} \Pi(x, k) \mathrm{d} k, z \in \mathbb{C} ;
$$

under proper regularity conditions about the payoff and the Fourier transform of the underlying dynamic variables (see e.g. [16]), it can be proved that

$$
\mathrm{e}^{r T} \Pi_{0}=\frac{1}{2 \pi} \int_{\mathrm{i} v-\infty}^{\mathrm{i} v+\infty} \mathrm{e}^{-\mathrm{i} z k} \mathbb{E}^{\mathbb{Q}}\left[\hat{\Pi}_{X(T)+\log \left(S_{0}\right)}(z)\right] \mathrm{d} z
$$

in some strip of $\mathbb{C}$. Let us consider the payoff functions $\Phi_{1}(x, k)=\left(\mathrm{e}^{k}-\mathrm{e}^{x}\right)^{+}$, and $\Phi_{2}(x, k)=\mathbb{I}_{\{x \leq k\}}$, in such a way $\Pi_{0}^{P}(\kappa, T)=\mathrm{e}^{-r T} \mathbb{E}^{\mathbb{Q}}\left[\Phi_{1}\left(X_{T}+\log \left(S_{0}\right), k\right)\right]$ and $\mathbb{P}\left\{S_{T} \leq v\right\}=\mathbb{P}\left\{X_{T} \leq k\right\}=\mathbb{E}\left[\Phi_{2}\left(X_{T}, k\right)\right]$, with $k=\log \left(v / S_{0}\right)$. Their GFT w.r.t. the trigger parameter $k$ are

$$
\hat{\Phi}_{1}(x, z)=\frac{\left.\mathrm{e}^{x(1+\mathrm{i} z}\right)}{\mathrm{i} z-\mathrm{z}^{2}}, \quad v>1 \text { and } \hat{\Phi}_{2}(x, z)=\frac{\mathrm{i}}{\mathrm{z}} \mathrm{e}^{\mathrm{i} x z}, \quad v>0
$$

respectively. Hence we get the formulas

$$
\begin{aligned}
& \Pi_{0}^{P}(k, T)=\mathrm{e}^{-r T} \frac{\mathrm{e}^{v k} S_{0}^{1-v}}{\pi} \\
& \cdot \mathfrak{R}\left(\int_{0}^{+\infty} \mathrm{e}^{-\mathrm{i} u\left(k-\log \left(S_{0}\right)\right)} \frac{\phi_{X}^{\mathbb{Q}}(u+\mathrm{i}(v-1))}{v^{2}-u^{2}-v+\mathrm{i} u(1-2 v)} \mathrm{d} u\right), v>1
\end{aligned}
$$


and

$$
\begin{aligned}
& \mathbb{P}\left\{S_{T} \leq v\right\}=\frac{\left(v / S_{0}\right)^{v}}{\pi} \mathfrak{R}\left(\int_{0}^{+\infty} \mathrm{e}^{-\mathrm{i} u \log \left(v / S_{0}\right)} \frac{\phi_{X}^{\mathbb{P}}(u+\mathrm{i} v)}{v-\mathrm{i} u} \mathrm{~d} u\right), \\
& v>0
\end{aligned}
$$

$\phi_{X}^{\mathbb{Q}, \mathbb{P}}(\mathrm{z})=\mathbb{E}^{\mathbb{Q}, \mathbb{P}}\left[\mathrm{e}^{\mathrm{i} Z X_{T}}\right]$ being the (generalized) Fourier transform, or characteristic function, of the random variable $X_{T}$ under the appropriate measure. If this is a regular functions in a properly defined strip of $\mathbb{C}$, the transform method can be applied in both cases (see [16]). Since under the Assumption (2.1) the optimality condition is

$$
\left(K-q_{1-\alpha}\left(S_{T}\right)\right)=\frac{\Pi_{0}^{P}(K, T)}{\frac{\partial}{\partial K} \Pi_{0}^{P}(K, T)} \equiv \frac{\Pi_{0}^{P}(K, T)}{\mathrm{e}^{-r T} \mathbb{Q}\left(S_{T} \leq K\right)} .
$$

the optimal hedging strategy is then implemented by running twice a root search algorithm to find the values

1) $q^{*}$ such that

$$
\begin{aligned}
& \frac{\left(q^{*} / S_{0}\right)^{\bar{v}}}{\pi} \mathfrak{R}\left(\int_{0}^{+\infty} \mathrm{e}^{-\mathrm{i} u \log \left(q^{*} / s_{0}\right)} \frac{\phi_{X}^{\mathbb{P}}(u+\mathrm{i} \bar{v})}{\bar{v}-\mathrm{i} u} \mathrm{~d} u\right)=\alpha, \\
& \bar{v}>0
\end{aligned}
$$

2) $K^{*}$ such that

$$
\begin{aligned}
& \left(K^{*}-q^{*}\right) \\
& =\frac{S_{0} \mathfrak{R}\left(\int_{0}^{+\infty} \mathrm{e}^{-\mathrm{i} u\left(\log \left(K^{*} / s_{0}\right)\right)} \frac{\phi_{X}^{\mathbb{Q}}(u+\mathrm{i}(v-1))}{v^{2}-u^{2}-v+\mathrm{i} u(1-2 v)} \mathrm{d} u\right)}{\mathfrak{R}\left(\int_{0}^{+\infty} \mathrm{e}^{-\mathrm{i} u\left(\log \left(K^{*} / s_{0}\right)\right)} \frac{\phi_{X}^{\mathbb{Q}}(u+\mathrm{i}(\bar{v}-1))}{\bar{v}-\mathrm{i} u} \mathrm{~d} u\right)}, \\
& v>1, \bar{v}>0 .
\end{aligned}
$$

Numerical quadrature must be used for integral evaluation. Alternatively the FFT algorithm can be used to efficiently approximate integrals (see [16]) and then a standard root-finding routine will find the required solutions.

\subsection{Some Numerical Results}

We report some numerical results about the valuation of the optimal hedging strategy in the regime-switching jump-diffusion framework. An extended set of results can be found in [17]. All numerical procedures were implemented in the $\mathrm{MatLab}^{\odot}$ framework. A standard rootsearch algorithm was used to solve Equations (19) and (20), with $v=2$ and $\bar{v}=1$, together with the GaussLobatto quadrature for approximating the corresponding integrals. Few milliseconds were needed to get the required quantities on an Intel ${ }^{\odot}$ Core i5.
We consider a two-state regime switching version of the jump-diffusion model with gaussian jumps $Y(i) \sim \mathcal{N}\left(a_{i}, b_{i}\right), i=1,2$, characterized by the parameters $\sigma_{i}, \lambda_{i}>0$ and $\mu_{i}, i=1,2$. The two state Markov chain $\alpha(t)$ has generator $Q=\left(\begin{array}{cc}-q_{1} & q_{1} \\ q_{2} & -q_{2}\end{array}\right)$.

The first issue we consider is the reduction of risk obtained by implementing the optimal hedging strategy in the RSJD framework. The risk reduction percentage $R=1-\frac{\operatorname{VaR}_{\alpha}\left(L_{0}^{h^{*}, K^{*}}\right)}{\operatorname{VaR}_{\alpha}\left(L^{u}\right)}$ evaluated for different set of parameters range from $4.39 \%$ up to $58 \%$, meaning that the strategy is effective in reducing the portfolio VaR, even in presence of jumps and regime-switching. On the other hand, by changing the value of some relevant parameters inside each model (GBM, JD, RSGBM, RSJD) the profile of the hedging portfolio $\mathrm{VaR}$ can change significantly. Hence we face the following issue: what is the effect of a wrong model specification which discards regime switchings and jumps, when they are indeed present in the market, and consider the simpler GBM model? In order to explore the model sensitivity of the optimal hedging strategy, we implemented the following exercise. We firstly fixed a RSJD model by choosing a complete set of parameters. Then we generated a set of call/put prices on which we calibrate the GBM model, finding the volatility $\hat{\sigma}_{G B M}$ with a constrained non-linear leastsquares algorithm: hence we run the optimal hedging strategy obtaining $K_{G B M}^{*}, h_{G B M}^{*}$ and correspondingly the minimal $\mathrm{VaR}, \mathrm{VaR}_{G B M}^{*}$. We finally calculated the probability

$$
\beta_{\text {RSJD }}=\mathbb{P}\left(L^{K^{*}, h^{*}} \geq \operatorname{VaR}_{G B M}^{*}\right)
$$

under the RSJD model. This step requires to evaluate the integral in (18): as before, we use a Gauss-Lobatto quadrature algorithm. Results are shown in Tables $\mathbf{1}$ and 2.

Table 1. Optimal hedging strategy $\left(K^{*}, h^{*}, V_{a R}^{*}\right)$ under the simulated true model (first column), the fitted GBM model (second column-estimated volatility) and the corresponding value of $\beta_{R S J D}$. Here $\sigma_{1}=0.3, \sigma_{2}=0.05$, $\lambda_{1}=2, \lambda_{2}=0.8, a_{1}=0.0, a_{2}=0.0, b_{1}=0.08, \quad b_{2}=0.15$, $q_{1}=1, q_{2}=0.2$; furthermore $r=0.5 \%, \alpha=0.01$ and the budget constraint is $C=0.1$.

\begin{tabular}{cccc}
\hline & $\begin{array}{c}\text { Optimal strategy } \\
\text { RSJD }\end{array}$ & $\begin{array}{c}\text { Optimal strategy } \\
\text { GBM }(\hat{\sigma})\end{array}$ & \multirow{2}{*}{$\beta_{\text {RSJD }}$} \\
\hline \multirow{2}{*}{$\mathrm{T}=0.5$} & $64.7442,0.7197$, & $65.6191,0.8433$, & \multirow{2}{*}{0.0132} \\
& 37.0189 & $35.3880(0.2905)$ & \\
$\mathrm{T}=1$ & $55.5928,0.6165$, & $57.1579,0.7644$, & \multirow{2}{*}{0.0148} \\
& 47.1767 & $44.4718(0.2689)$ & \\
$\mathrm{T}=3$ & $41.6851,0.5294$, & $43.5664,0.7382$, & \multirow{2}{*}{0.0157} \\
& 62.3356 & $58.6379(0.2254)$ & \\
\hline
\end{tabular}


Table 2. Optimal hedging strategy $\left(K^{*}, h^{*}, \mathrm{VaR}^{*}\right)$ under the simulated true model (first column), the fitted GBM model (second column-estimated volatility) and the corresponding value of $\beta_{R S J D}$. Here $\sigma_{1}=0.3, \sigma_{2}=0.05$, $\lambda_{1}=2, \lambda_{2}=0.8, a_{1}=0.05, a_{2}=-0.3, b_{1}=0.08$, $b_{2}=0.15, q_{1}=1, q_{2}=0.2 ;$ furthermore $r=0.5 \%$, $\alpha=0.01$ and the budget constraint is $C=0.01$.

\begin{tabular}{|c|c|c|c|}
\hline & $\begin{array}{c}\text { Optimal strategy } \\
\text { RSJD }\end{array}$ & $\begin{array}{l}\text { Optimal strategy } \\
\text { GBM }(\hat{\sigma})\end{array}$ & $\beta_{R S I D}$ \\
\hline $\mathrm{T}=0.5$ & $\begin{array}{c}\text { 61.1841, } 0.0581 \\
45.2341\end{array}$ & $\begin{array}{l}63.3076,0.0816, \\
41.3746(0.3137)\end{array}$ & 0.0166 \\
\hline $\mathrm{T}=1$ & $\begin{array}{c}45.8347,0.0833 \\
60.5069\end{array}$ & $\begin{array}{l}52.7089,0.0744, \\
52.6106(0.3047)\end{array}$ & 0.0255 \\
\hline $\mathrm{T}=3$ & $\begin{array}{c}18.8056,0.4015 \\
83.7630\end{array}$ & $\begin{array}{l}32.7103,0.0797 \\
72.5986(0.2930)\end{array}$ & 0.0640 \\
\hline
\end{tabular}

Table 3. Optimal hedging strategy $\left(K^{*}, h^{*}, \mathrm{VaR}^{*}\right)$ under the simulated true model (first column), the fitted GBM model (second column-estimated volatility) and the corresponding value of $\beta_{R S J D}$. Here $\sigma_{1}=0.27, \sigma_{2}=0.13$, $\lambda_{1}=6.8, \lambda_{2}=0.8, a_{1}=-0.13, a_{2}=-0.34, b_{1}=0.08$, $b_{2}=0.15, q_{1}=6.5, q_{2}=0.002$; furthermore $r=0.5 \%$, $\alpha=0.01$ and the budget constraint is $C=0.01$.

\begin{tabular}{cccc}
\hline & $\begin{array}{c}\text { Optimal strategy } \\
\text { RSJD }\end{array}$ & $\begin{array}{c}\text { Optimal strategy } \\
\text { GBM }(\hat{\sigma})\end{array}$ & $\beta_{\text {RSJD }}$ \\
\hline $\mathrm{T}=0.5$ & $\begin{array}{c}38.3721,0.2497, \\
\text { 66.0564 }\end{array}$ & $60.0168,0.0785$, & \multirow{2}{*}{0.1165} \\
$\mathrm{~T}=1$ & $26.6034,0.4103$, & $49.4859,0.0737$, & \\
& 76.3270 & $55.8926(0.3320)$ & 0.1304 \\
$\mathrm{~T}=1.5$ & $19.6884,0.6506$, & $41.9632,0.0741$, & \multirow{2}{*}{0.1430} \\
& 81.8069 & $63.4963(0.3291)$ & \\
\hline
\end{tabular}

Notice that even when the optimal strategies are similar, the probability that the portfolio loss exceeds the (optimal) VaR is greater than the fixed level $\alpha=0.01$. Of course this behavior depends on the choice of the parameters, but the underestimation produced by a wrong model choice can be quite severe: see Table 3, where parameters from a real data set were used (see [8]).

\section{REFERENCES}

[1] D. H. Ahn, J. Boudoukh, M. Richardson and R. F. Whitelaw, "Optimal Risk Management Using Options," Journal of Finance, Vol. 54, No. 1, 1999, pp. 359-375. doi:10.1111/0022-1082.00108

[2] J. Annaert, G. Deelstra, D. Heyman and M. Vanmaele, "Risk Management of a Bond Portfolio Using Options," Insurance: Mathematics and Economics, Vol. 41, No. 3, 2007, pp. 299-316. doi:10.1016/i.insmatheco.2006.11.002

[3] F. Antonelli, A. Ramponi and S. Scarlatti, "Option Based
Risk Management of a Bond Portfolio under Regime Switching Interest Rates," Decisions in Economics and Finance, 2011. doi:10.1007/s10203-011-0123-1

[4] J. Hamilton, "A New Approach to the Economic Analysis of Non Stationary Time Series and the Business Cycle," Econometrica, Vol. 57, No. 2, 1989, pp. 357-384. doi:10.2307/1912559

[5] R. Cont and P. Tankov, "Financial Modelling with Jump Processes," Chapman \& Hall, CRC Press, Boca Raton, 2003. doi: $10.1201 / 9780203485217$

[6] R. Kawata and M. Kijima, "Value at Risk in a Market Subject to Regime Switching," Quantitative Finance, Vol. 7, No. 6, 2007, pp. 609-619. doi:10.1080/14697680601161795

[7] A. Taamouti, "Analytical Value-at-Risk and Expected Shortfall under Regime-Switching," Finance Research Letters, Vol. 6, No. 3, 2009, pp. 138-151. doi:10.1016/j.frl.2009.03.004

[8] A. Ramponi, "On Fourier Transform Methods for Regime-Switching Jump-Diffusions and the Pricing of Forward Starting Options," International Journal of Theoretical and Appplied Finance, Vol. 15, No. 5, 2012, 26 p.

[9] D. Duffie and J. Pan, "Analytical Value-at-Risk with Jumps and Credit Risk," Finance and Stochastics, Vol. 5, No. 2, 2001, pp. 155-180. doi:10.1007/PL00013531

[10] O. Le Courtois and C. P. Walter, "A Study on Value-atRisk and Lévy Processes,” 2009. http://ssrn.com/abstract=1598360 doi: $10.2139 /$ ssrn. 1598360

[11] Y. S. Kim, S. T. Rachev, M. L. Bianchi and F. J. Fabozzi, "Computing VaR and AVaR in Infinitely Divisible Distributions," Probability and Mathematical Statistics, Vol. 30, No. 2, 2010, pp. 223-245.

[12] M. Scherer, S. T. Rachev, Y. S. Kim and F. J. Fabozzi, “A FFT-Based Approximation of Tempered Stable and Tempered Infinitely Divisible Distributions," 2009.

http://www.ams.sunysb.edu/ rachev/publication/Standard TSDistr_20100306final.pdf

[13] P. Artzner, F. Delbaen, J. Eber and D. Heath, "Coherent Measures of Risk," Mathematical Finance, Vol. 9, No. 3, 1999, pp. 203-228. doi:10.1111/1467-9965.00068

[14] W. J. Runggaldier, "Jump-Diffusion Models," In: S.T. Rachev, Ed., Handbook of Heavy Tailed Distributions in Finance, Elesevier/North-Holland, Amsterdam, 2003, pp. 169-209.

[15] P. Carr and D. B. Madan, "Option Valuation Using the Fast Fourier Transform," Journal of Computational Finance, Vol. 2, No. 4, 1999, pp. 61-73.

[16] R. W. Lee, "Option Pricing by Transform Methods: Extensions, Unifications and Error Control," Journal of Computational Finance, Vol. 7, No. 3, 2004, pp. 51-86.

[17] A. Ramponi, “Computing Quantiles in Regime-Switching Jump-Diffusions with Application to Optimal Risk Management: A Fourier Transform Approach," arXiv: 1207.6759, 2012. 